\title{
Spin-Lattice Relaxation in Metal-Organic Platinum(II) Complexes
}

\author{
H. H. H. Homeier ${ }^{1,2}$, J. Strasser, and H. Yersin ${ }^{3}$ \\ Institut für Physikalische und Theoretische Chemie, \\ D-93040 Regensburg, Germany
}

\begin{abstract}
The dynamics of spin-lattice relaxation (slr) of metal-organic Pt(II) compounds is studied. Often, such systems are characterized by pronounced zero-field splittings (zfs) of the lowest-lying triplets. Previous expressions for the Orbach slr process do not allow to treat such splitting patterns properly. We discuss the behavior of a modified Orbach expression for a model system and present results of a fit of the temperature dependence of the spin-lattice relaxation rate of $\mathrm{Pt}(2 \text {-thpy })_{2}$ based on the modified expression.
\end{abstract}

Key words: Metal-organic Platinum(II) complexes, Shpol'skii matrices, Spin-lattice relaxation, Orbach process, Raman process, Direct process, Triplets, Zero-field splittings

Transition metal complexes with organic chelate ligands and their lowest excited states are of potential use for solar energy conversion [1-7]. Recently, the processes of spin-lattice relaxation and the decay behavior of excited states have been studied experimentally for such systems in Shpol'skii matrices. [814] Of special importance are compounds with a Pt(II) central ion. Pt(II) systems exhibit many different types of low-lying excited triplets that include metal-centered (MC) dd* states [15,16], metal-to-ligand-charge-transfer (MLCT) states [17-19], intra-ligand-charge-transfer (ILCT) states [10,13,20], ligand-ligand'-charge-transfer (LL'CT) states [6,7], and ligand-centered (LC)

1 Author for correspondence. Address: PD Dr. H. H. H. Homeier, Institut für Physikalische und Theoretische Chemie, Universität Regensburg, D-93040 Regensburg, Germany. FAX: +49-941-943 4719. Email: Herbert.Homeier@na-net.ornl.gov

2 WWW: http://www.chemie.uni-regensburg.de/ hoh05008

3 Author for correspondence. Address: Prof. Dr. H. Yersin, Institut für Physikalische und Theoretische Chemie, Universität Regensburg, D-93040 Regensburg, Germany. FAX: +49-941-943 4488. Email: Hartmut.Yersin@chemie.uni-regensburg.de 
Table 1

Electronic origins $\mathrm{E}\left[\mathrm{cm}^{-1}\right]$ (lowest triplet sublevel of $\mathrm{T}_{1}$, lowest site), zero-field splittings $\left[\mathrm{cm}^{-1}\right]\left(\Delta E_{b a}\right.$ : Energy difference between $|b\rangle$ and $|a\rangle, \Delta E_{c b}$ : Energy difference between $|c\rangle$ and $|b\rangle$ ), spin-lattice relaxation times $\tau_{\text {slr }}$ [ns] at $1.2 \mathrm{~K}$, and transition types for various $\mathrm{Pt}(\mathrm{II})$ complexes with organic ligands

\begin{tabular}{|c|c|c|c|c|c|c|}
\hline Complex & $\mathrm{E}$ & $\Delta E_{b a}$ & $\Delta E_{c b}$ & $\tau_{\text {slr }}$ & Type & Ref. \\
\hline $\operatorname{Pt}(2 \text {-thpy })_{2}{ }^{a)}$ & 17156 & 7 & 9 & 710 & $\mathrm{LC} / \mathrm{MLCT}$ & {$[9,21,14]$} \\
\hline $\operatorname{Pt}(2-$ thpy $\left.)(\mathrm{CO}) \mathrm{Cl}^{a}\right)$ & 18012 & 0.055 & 3.8 & 3000 & $\mathrm{LC} / \mathrm{MLCT}$ & {$[14,24]$} \\
\hline $\operatorname{Pt}(\text { phpy })_{2}{ }^{a)}$ & 19571 & 6.9 & 25.1 & 390 & $\mathrm{LC} / \mathrm{MLCT}$ & {$[14]$} \\
\hline $\left.\operatorname{Pt}(3 \text {-thpy })_{2}{ }^{a}\right)$ & 18020 & 13 & 9 & $\approx 25$ & $\mathrm{LC} / \mathrm{MLCT}$ & {$[25,26]$} \\
\hline$\left[\mathrm{Pt}(\mathrm{bpy})_{2}\right]\left(\mathrm{ClO}_{4}\right)_{2}{ }^{b)}$ & 21237 & $<1$ & $<1$ & $>50 \cdot 10^{3}$ & $\mathrm{LC} / \mathrm{MC}$ & {$[23]$} \\
\hline $\operatorname{Pt}(\mathrm{qol})_{2}{ }^{a)}$ & 15426 & $<1$ & $<1$ & $>60 \cdot 10^{3}$ & ILCT & {$[10,13]$} \\
\hline $\operatorname{Pt}(\mathrm{qtl})_{2}{ }^{a)}$ & 13158 & $<1$ & $<1$ & $>7000$ & ILCT & {$[13]$} \\
\hline $\left.\mathrm{Pt}(\mathrm{phpy})(\mathrm{CO}) \mathrm{Cl}^{a}\right)$ & 20916 & $<1$ & 6.4 & & $\mathrm{LC} / \mathrm{MLCT}$ & {$[27]$} \\
\hline $\operatorname{Pt}(\mathrm{bhq})_{2}{ }^{c)}$ & 19814 & 11 & 28 & & LC/MLCT & {$[28]$} \\
\hline $\operatorname{Pt}(\mathrm{phpz})_{2}^{a)}$ & 22952 & 9 & 7 & & LC/MLCT & {$[25]$} \\
\hline
\end{tabular}
a) In n-octane
b) Neat material
c) In n-decane

states with some MLCT and/or MC contribution [21-23]. In the following, we focus to $\mathrm{Pt}(\mathrm{II})$ systems with heterocyclic chelate ligands.

As shown in Tab. 1, the low-lying triplets of these systems are characterized by a rather large variation of zero-field splittings (zfs) in the range from less than $0.1 \mathrm{~cm}^{-1}$ to about $40 \mathrm{~cm}^{-1}$. The larger splittings are mainly due to spin-orbit coupling. For the same complex in different matrices, the lowest triplet states are shifted in energy (in many cases in the range of $200-400$ $\mathrm{cm}^{-1}$ ). The corresponding optical spectra show rich vibrational structure that may be well resolved (about $2 \mathrm{~cm}^{-1}$ ) by choosing appropriate matrices and by employing methods of emission and/or excitation line narrowing.

At low temperatures (several Kelvin), the processes of spin-lattice relaxation occurring between the triplet sublevels $|a\rangle,|b\rangle$, and $|c\rangle$ are relatively slow with relaxation times as long as hundreds of nano-seconds and even up to many micro-seconds (See Tab. 1 and Refs. [9-14]) due to the low density of phonon states corresponding to such zfs patterns.

To discuss these processes, we assume that the perturbation $V$ caused by the phonons couples the electronic states of the chromophore essentially linearly 


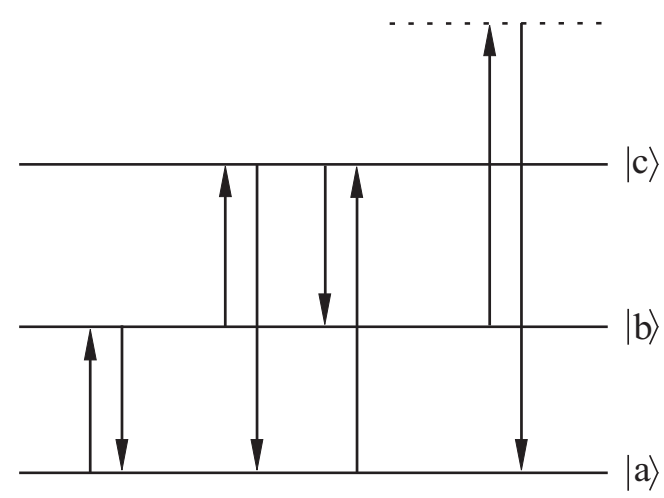

(a) (b) (c)

Fig. 1. Processes of spin-lattice relaxation: a) Direct process. b) Orbach process. c) Raman process.

(e.g. see Ref. [29, p. 228])

$$
V=V_{1} \sum_{k} \epsilon_{k}+\ldots
$$

where $\epsilon_{k}$ is the strain corresponding to the phonon mode with wave vector $k$ in the long wavelength limit. The matrix elements of $V_{1}$ are denoted by $V_{b a}=$ $\left|\left\langle b\left|V_{1}\right| a\right\rangle\right|$ and analogous expressions for $V_{c a}$ and $V_{c b}$. The energy differences are $\Delta E_{b a}$ between $|b\rangle$ and $|a\rangle, \Delta E_{c b}$ between $|c\rangle$ and $|b\rangle$, and $\Delta E_{c a}$ between $|c\rangle$ and $|a\rangle$. The usual notation $\beta=1 /\left(k_{B} T\right)$ for given temperature $T$ and Boltzmann constant $k_{B}$, and the abbreviations $C_{b a}=C V_{b a}^{2}\left(\Delta E_{b a}\right)^{3}$ and analogous ones for $C_{c a}$ and $C_{c b}$ are also used. Here, the parameter $C=3 /\left(2 \pi \hbar^{4} \rho v^{5}\right)$ is defined in terms of mass density $\rho$ and (average) velocity $v$ of sound of the matrix. The $\left(\Delta E_{b a}\right)^{3}$ dependence of $C_{b a}$ should be kept in mind.

The following relaxation processes (see Fig. 1) occur:

Direct process: The rate is given by [30, p. 541], [29, p. 229]

$$
\begin{aligned}
k_{a, b}^{(\text {direct })} & =k_{a b}+k_{b a} \\
& =C_{b a} \operatorname{coth}\left(\beta \Delta E_{b a} / 2\right) .
\end{aligned}
$$

Here, $k_{a b}$ and $k_{b a}$ are the rate constants for the up and down processes, respectively, given by the expressions

$$
\begin{aligned}
& k_{a b}=C_{b a} \frac{1}{\exp \left(\beta \Delta E_{b a}\right)-1}, \\
& k_{b a}=C_{b a} \frac{\exp \left(\beta \Delta E_{b a}\right)}{\exp \left(\beta \Delta E_{b a}\right)-1} .
\end{aligned}
$$


Analogous expressions hold for the up and down rates $k_{b c}, k_{c b}, k_{a c}$, and $k_{c a}$.

Orbach process: The rate for this process vanishes for $T \rightarrow 0 \mathrm{~K}$ exponentially. It depends on the splitting pattern of the three involved states: If the energy separation $\Delta E_{b a}$ of the two lower states $|a\rangle$ and $|b\rangle$ is much smaller than both the energy separations $\Delta E_{c a}$ and $\Delta E_{c b}$ to the upper state $|c\rangle$, then the well-known expression

$$
k_{a, b}^{(\text {Orbach })}=\frac{2 C_{c b} C_{c a}}{\left(C_{c a}+C_{c b}\right)} \frac{1}{\left(\mathrm{e}^{\beta \Delta E}-1\right)}
$$

holds approximately for low $T$. This original Orbach expression is derived under the assumption that the energy differences are given by $\Delta E=\Delta E_{c a}=$ $\Delta E_{c b}>0$. For a more general zfs pattern, the rate is given by the low-temperature approximation [31]

$$
k_{a, b}^{(\text {Orbach })}=\frac{k_{a c} k_{c b}+k_{b c} k_{c a}-k_{b c} k_{b a}}{k_{c a}+k_{c b}-k_{b a}}
$$

with up and down rates as given in Eq. (3). The modified expression (5) contains Eq. (4) as a limiting case (see Ref. [31]).

Raman process: For low temperature, the rate may be approximated by

$$
k_{a, b}^{(\operatorname{Raman})}=D T^{n}
$$

with a constant $D$ and $n=5$ for non-Kramers ions [32]. In the cases under study, this $T^{5}$ dependence fits the experimental observations [31] better than the $T^{7}$ dependence observed in other systems.

The relative importance of the various slr processes is largely dependent on the size of the zfs and the energy separations to further electronic states. For instance, in systems like $\mathrm{Pt}(\mathrm{qol})_{2}$ and $\mathrm{Pt}(\mathrm{qtl})_{2}$ with a very small total zfs (see Tab. 1) and no further electronic states in the vicinity of $\mathrm{T}_{1}$, direct and Orbach processes are expected to be very small due to the $\Delta E^{3}$ dependence of these processes, and the Raman process is expected to dominate. Compare also Ref. [31].

The behavior of the above expressions is illustrated for a model system (without a Raman process) and with parameters $\Delta E_{b a}=\Delta E_{c b}=7 \mathrm{~cm}^{-1}, v=2000$ $\mathrm{m} / \mathrm{s}, \rho=1.1 \mathrm{~g} / \mathrm{cm}^{3}, V_{b c}=10 \mathrm{~cm}^{-1}, V_{a c}=20 \mathrm{~cm}^{-1}, V_{a b}=3 \mathrm{~cm}^{-1}$. In Fig. 2, the relative errors of the approximations for both direct and Orbach process, i.e., 


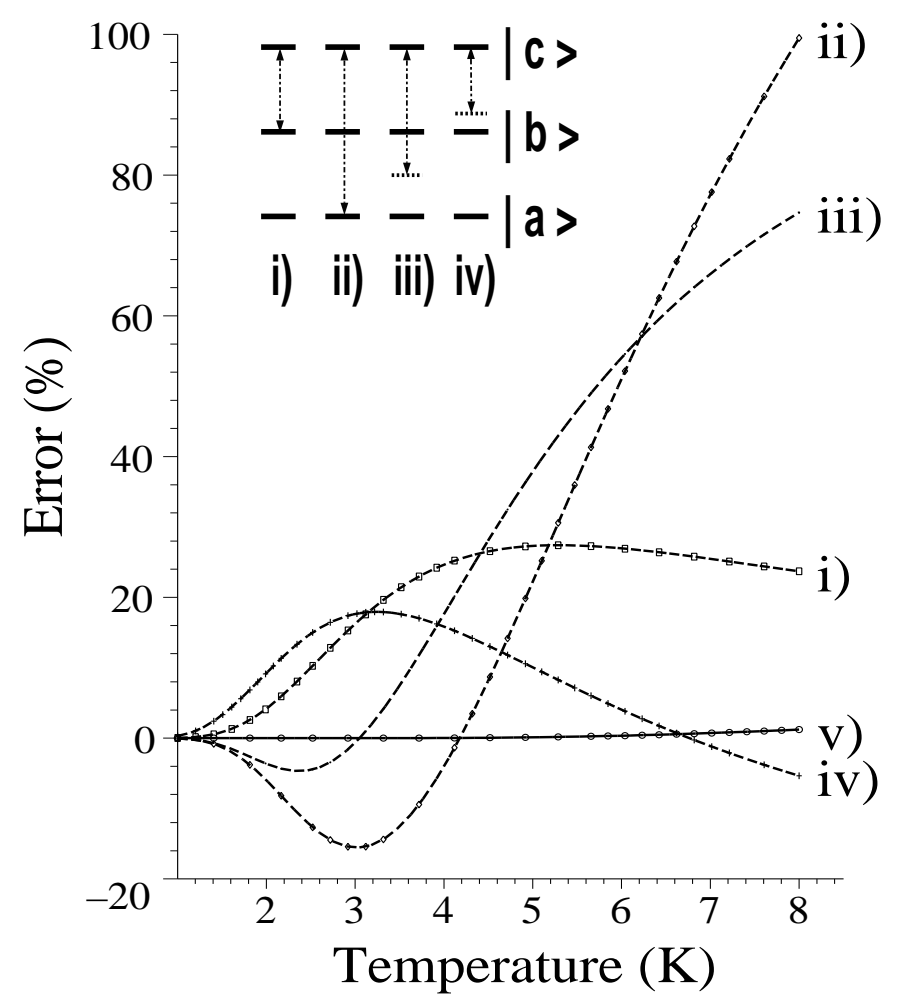

Fig. 2. Relative errors of the relaxation rate expressions with respect to Eq. (7) as a function of temperature $T$. Plotted are the errors of $k_{a, b}^{(\text {direct })}+k_{a, b}^{(\text {Orbach })}$ with Eq. (2) for the direct process in combination with the original Orbach expression (4) for different values of $\Delta E$ ( i) $\Delta E=\Delta E_{c b}=7 \mathrm{~cm}^{-1}$, ii) $\Delta E=\Delta E_{c a}=14 \mathrm{~cm}^{-1}$, iii) $\Delta E=\left(\Delta E_{c a}+\Delta E_{c b}\right) / 2=10.5 \mathrm{~cm}^{-1}$, iv) $\left.\Delta E=\Delta E_{\text {fit }}=5.4 \mathrm{~cm}^{-1}\right)$ and v) with the modified expression (5).

for the sum $k_{a, b}^{(\text {direct })}+k_{a, b}^{(\text {Orbach })}$ as obtained using Eq. (2) in combination either with Eq. (4) or Eq. (5), respectively. The errors are calculated with respect to the exact rate

$$
\begin{aligned}
& k_{a, b}^{(\text {Orbach }+ \text { direct })} \\
& =\frac{1}{2}\left(k_{b c}+k_{a c}+k_{c b}+k_{c a}+k_{b a}+k_{a b}\right) \\
& -\frac{1}{2}\left(\left(k_{b c}+k_{c b}-k_{a b}-k_{c a}-k_{a c}+k_{b a}\right)^{2}\right. \\
& \left.+4\left(k_{c b} k_{c a}-k_{a b} k_{c a}-k_{b a} k_{c b}+k_{b a} k_{a b}\right)\right)^{1 / 2}
\end{aligned}
$$

for the three-level system that is obtained from the rate equations [31].

Applying the original Orbach expression, i.e., using Eq. (4) in combination with (2) for the direct process, the prefactor $2 C_{c b} C_{c a} /\left(C_{c a}+C_{c b}\right)$ was computed from the model parameters, but different values of the parameter $\Delta E$ have been used: $\Delta E=\Delta E_{c b}$ corresponds to using the minimum distance of 
state $|c\rangle$ to the states $|a\rangle$ and $|b\rangle$ (curve i) in Fig. 2), $\Delta E=\Delta E_{c a}$ corresponds to using the maximum distance (curve ii)), and $\Delta E=\left(\Delta E_{c a}+\Delta E_{c b}\right) / 2$ corresponds to using the mean distance (curve iii)). The value $\Delta E=\Delta E_{\text {fit }}=$ $5.4 \mathrm{~cm}^{-1}$ is obtained by a least square fit of the exact data with one fit parameter $\Delta E$ (curve iv)), i.e., for the direct process and the prefactor of Eq. (4), the exact expressions were used during the fit. Interestingly, $\Delta E_{\text {fit }}$ is less than any of the other differences of the energies. Alternatively, one could try to use the prefactor in Eq. (4) as an additional fit parameter. But then, one cannot hope to extract the model values of $C_{c b}$ and $C_{c a}$ from such a fit. Finally, curve v) in Fig. 2 was obtained using the modified expression (5) in combination with Eq. (2) for the direct process. Clearly, the modified approach yields much reduced errors over a large temperature range. Thus, Orbach's original expression (4) that was designed for a different pattern of the energy levels cannot be applied to a pattern with $\Delta E_{c b} \approx \Delta E_{b a}$ for any reasonable choice of the parameter $\Delta E$.

We remark that similar results are also obtained for different choices of the parameters. For instance, for a value of $v$ smaller by a factor $f$, the same results for the absolute rates would be obtained, if all the matrix elements of $V_{1}$ are also chosen smaller by a factor $f^{5 / 2}$, e.g., for $v=1500 \mathrm{~m} / \mathrm{s}$ and $V_{b c}=4.87 \mathrm{~cm}^{-1}, V_{a c}=9.74 \mathrm{~cm}^{-1}, V_{a b}=1.46 \mathrm{~cm}^{-1}$. Moreover, fixing all the other parameters, any rescaling of the three matrix elements by an arbitrary common positive factor yields the same error curves since we are dealing with relative errors and, under this scaling, all up and down rates $k_{a b}, k_{b a}$ etc., and, hence, all slr rates in the model are multiplied by a common factor.

It is of interest to present an example of the application of the above formalism to the spin-lattice relaxation observed for the lowest triplet of the $\operatorname{Pt}(2 \text {-thpy })_{2}$ complex in an n-octane matrix. This compound is depicted in of Fig. 3, and some properties are collected in Table 1. The experimental spin-lattice relaxation rate $k^{(s l r)}$ is obtained from the measured emission decay rate of state $|b\rangle$ by subtraction of the corresponding triplet deactivation rate to the ground state [31]. For the fit, we used Eq. (2) for the direct process, the modified expression (5) for the Orbach process, and Eq. (6) with $n=5$ for the Raman process, i.e., for a $T^{5}$ low temperature dependence. As prefactor of the direct process, we used the low temperature limit of $k^{(s l r)}$. The ratio of $C_{c a} / C_{c b}$ can be obtained independently from time-resolved excitation spectra [9,31]. Also, all energy separations $\Delta E_{b a}$ and $\Delta E_{c b}$ are available from highly resolved spectra $[9,21,14,31]$. Thus, as fit parameter, only the prefactor $D$ of the Raman process and the constant $C_{c a}$ remain. For such a two-parameter fit as displayed in Fig. 3, the result is highly satisfactory.

A three-parameter fit based on the original Orbach expression (4) using the parameters $D, \Delta E$ and the prefactor in Eq. (4) yields the value $\Delta E=11.4 \mathrm{~cm}^{-1}$ (and a nearly doubled prefactor $D$ for the Raman process in comparison to 


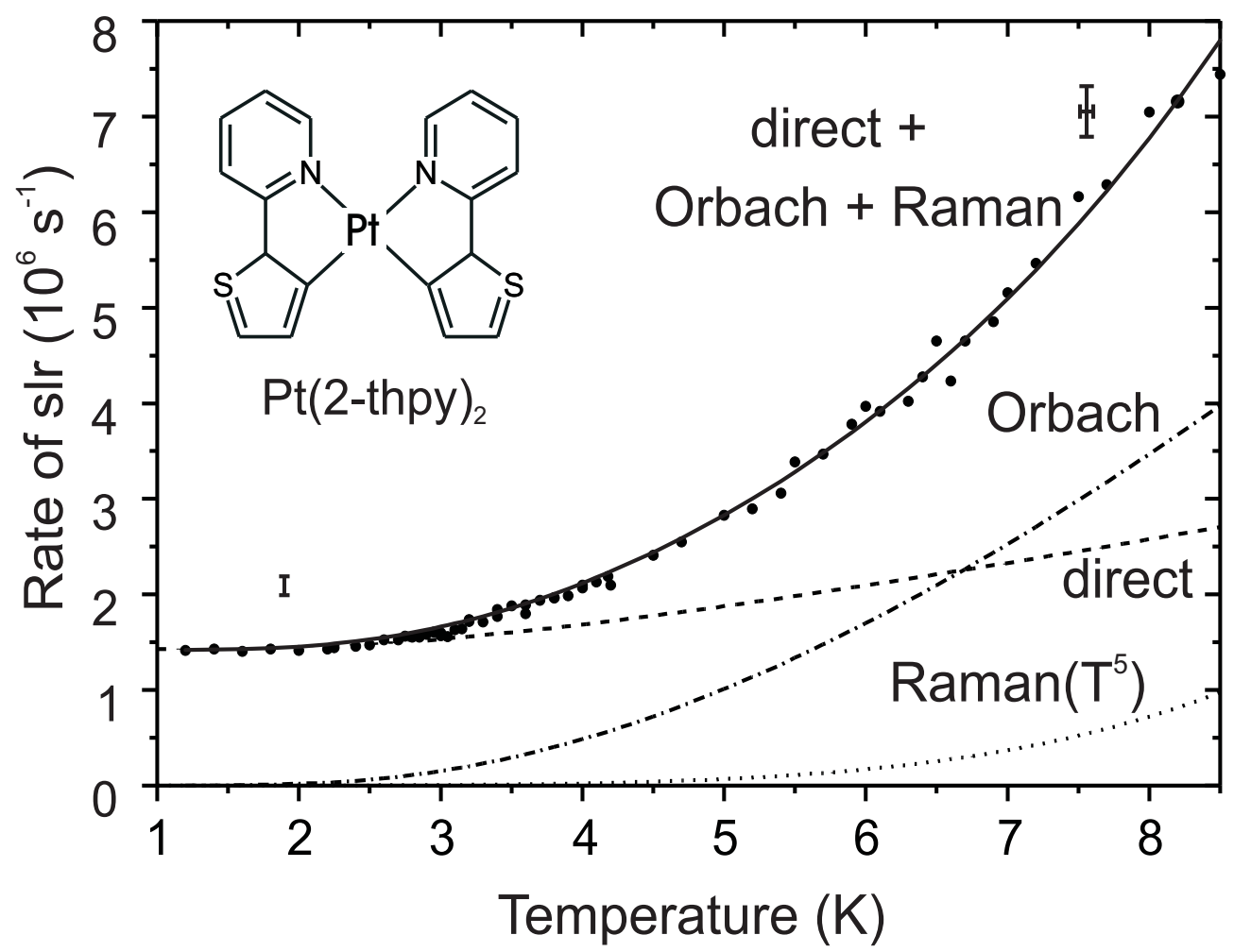

Fig. 3. Fit of the spin-lattice relaxation rate $k^{(s l r)}$ as a function of temperature for $\operatorname{Pt}(2 \text {-thpy })_{2}$ in an n-octane matrix. Displayed are the contributions of the direct process (Eq. (2)), the Orbach process (using the modified expression (5)), and the Raman $T^{5}$ process (Eq. (6)).

the fit displayed in Fig. 3). A similar value for $\Delta E$ was obtained in Ref. [9] by a somewhat different fitting procedure. Both these values are unphysical since they do not correspond to any of the observed energy differences (see Tab. 1). We remark that the present study was triggered by this difficulty of using the original Orbach expression (4).

This result shows, as further ones presented in [33,31], that the use of the modified expression (5) for the Orbach process is necessary for a detailed understanding of the dynamics of the spin-lattice relaxation for low-lying triplets of metal-organic transition metal compounds with their characteristic patterns of zero-field splitting. Thus, although the present study concentrated on Pt(II) compounds, the result should be applicable to a more general class of compounds, namely, the whole platinum metal group complexes (compare, e.g., the recent results $[8,9]$ for $\left.\left[\mathrm{Ru}(\mathrm{bpy})_{3}\right]^{2+}\right)$.

Financial support by the Deutsche Forschungsgemeinschaft and the Fonds der Chemischen Industrie is gratefully acknowledged. 


\section{References}

[1] J. S. Conolly (Ed.), Photochemical Conversion and Storage of Solar Energy, Academic Press, New York, 1981.

[2] A. Harriman, M. A. West (Eds.), Photogeneration of Hydrogen, Academic Press, London, 1982.

[3] G. Calzaferri (Ed.), Proceedings of the $10^{\text {th }}$ International Conference on Photochemical Transformation and Storage of Solar Energy, volume 38 of Solar Energy Materials and Solar Cells, Interlaken, 1994.

[4] B. O’Regan, M. Grätzel, Nature 353 (1991) 737.

[5] A. Juris, V. Balzani, F. Barigelletti, S. Campagna, P. Belser, A. von Zelewsky, Coord. Chem. Rev. 84 (1988) 85.

[6] A. Vogler, H. Kunkely, J. Am. Chem. Soc. 103 (1981) 1559.

[7] S. D. Cummings, R. Eisenberg, J. Am. Chem. Soc. 118 (1996) 1949.

[8] H. Yersin, W. Humbs, J. Strasser, in: H. Yersin (Ed.), Electronic and Vibronic Spectra of Transition Metal Complexes, Vol. II, volume 191 of Topics in Current Chemistry, Springer-Verlag, Berlin, 1997, p. 153.

[9] J. Schmidt, J. Strasser, H. Yersin, Inorg. Chem. 36 (1997) 3957.

[10] D. Donges, J. K. Nagle, H. Yersin, Inorg. Chem. 36 (1997) 3040.

[11] H. Yersin, J. Strasser, J. Luminescence 72-74 (1997) 462.

[12] H. Yersin, D. Braun, Coord. Chem. Rev. 111 (1991) 39.

[13] D. Donges, J. K. Nagle, H. Yersin, J. Luminescence 72-74 (1997) 658.

[14] J. Strasser, D. Donges, W. Humbs, M. V. Kulikova, K. P. Balashev, H. Yersin, J. Luminescence 76-77 (1998) 611.

[15] L. G. Vanquickenborne, A. Ceulemans, Inorg. Chem. 20 (1981) 796.

[16] H. Yersin, H. Otto, J. I. Zink, G. Gliemann, J. Am. Chem. Soc. 102 (1980) 951.

[17] C. D. Cowman, H. B. Gray, Inorg. Chem. 15 (1976) 2823.

[18] H. Yersin, G. Gliemann, Ann. N.Y. Acad. Sci. 313 (1978) 539.

[19] G. Gliemann, H. Yersin, Struct. Bond. (Berlin) 62 (1985) 87.

[20] R. Ballardini, G. Varani, M. T. Indelli, F. Scandola, Inorg. Chem. 25 (1986) 3858 .

[21] H. Wiedenhofer, S. Schützenmeier, A. von Zelewsky, H. Yersin, J. Phys. Chem. 99 (1995) 13385. 
[22] M. Maestri, V. Balzani, C. Deuschl-Cornioley, A. von Zelewsky, Adv. Photochem. 17 (1992) 1.

[23] W. Humbs, H. Yersin, Inorg. Chim. Acta 265 (1997) 139.

[24] M. Glasbeek, W. Humbs, H. Yersin, unpublished results.

[25] H. Wiedenhofer, Ph.D. Thesis (in German), Universität Regensburg, Regensburg, Germany, 1994.

[26] M. Eichenseer, Diploma Thesis (in German), Universität Regensburg, Regensburg, Germany, 1999.

[27] D. Donges, Ph.D. Thesis (in German), Universität Regensburg, Regensburg, Germany, 1997.

[28] H. Backert, H. Yersin, A. von Zelewsky, in: $13^{\text {th }}$ International Symposium on Photochemistry and Photophysics of Coordination Compounds, Lipari, Italy, 1999, p. 90.

[29] B. Henderson, G. F. Imbusch, Optical Spectroscopy of Inorganic Solids, Clarendon Press, Oxford, 1989.

[30] A. Abragam, B. Bleaney, Electron Paramagnetic Resonance of Transition Ions, Clarendon Press, Oxford, 1970.

[31] J. Strasser, H. H. H. Homeier, H. Yersin, Chem. Phys. (in preparation).

[32] M. B. Walker, Can. J. Phys. 46 (1968) 1347.

[33] J. Strasser, Ph.D. Thesis (in German), Universität Regensburg, Regensburg, Germany, 1999. 